PROCEEDINGS OF THE

AMERICAN MATHEMATICAL SOCIETY

Volume 130, Number 9, Pages 2809-2810

S 0002-9939(02)06684-

Article electronically published on April 16, 2002

\title{
ERRATUM TO "A RELATION BETWEEN HOCHSCHILD HOMOLOGY AND COHOMOLOGY FOR GORENSTEIN RINGS"
}

\author{
MICHEL VAN DEN BERGH
}

(Communicated by Lance W. Small)

The paper [5] contains an error in the sense that Theorem 1 (the "duality theorem") is false in the generality stated. As a result the same is true for its corollaries: Proposition 3 and Corollary 6. The main conclusion, which is an affirmative answer to a question by Patrick Polo, remains valid however (see below).

That Theorem 1 is false as stated was pointed out in [2]. In general it can be seen as follows. If the conclusion of Theorem 1 is true, then the Hochschild dimension (the cohomological dimension of $H H^{*}$ ) of the ring $A$ is finite. So Theorem 1 must be false for every ring of infinite Hochschild dimension, and hence in particular for every ring of infinite global dimension.

Thus to save Theorem 1 we must assume that $A$ has finite Hochschild dimension (let us say that $A$ is smooth in this case). It is easy to see that in that case the proof becomes valid. The smoothness hypothesis is automatically satisfied in Proposition 2 (see [4) but it must be added in Proposition 3 and Corollary 6.

The following lemma shows that smoothness is a reasonable condition.

Lemma. (1) If $A$ is commutative of finite type over the ground field $k$, then $A$ is smooth in the above sense if and only it is smooth in the classical sense (the "only if" part is related to the Hochschild-Kostant-Rosenberg theorem).

(2) If $A$ and $B$ are smooth, then so is $A \otimes_{k} B$ (this is [1. Prop. 2(2)]).

(3) The following are equivalent:

(a) $A$ is smooth.

(b) $A^{e}$ has finite global dimension.

Remark. If $A$ and $B$ have finite global dimension, then this is not necessarily the case for $A \otimes_{k} B$. The standard counterexample is given by two fields of infinite transcendence degree.

Remark. In practice $A$ will often be a DG-algebra or an $A_{\infty}$-algebra. In this case the correct notion of smoothness is that $A$ should be a compact object in $D\left(A^{e}\right)$ $\left(\operatorname{Hom}_{D\left(A^{e}\right)}(A,-)\right.$ should commute with direct sums). The author learnt this from a talk by Kontsevich.

It follows that in order to answer Patrick Polo's question we need to show additionally that if $A$ is a regular minimal quotient of a semi-simple enveloping algebra

Received by the editors December 5, 2001.

1991 Mathematics Subject Classification. Primary 16E40.

Key words and phrases. Hochschild homology, Gorenstein rings.

The author is a senior researcher at the FWO. 
$U(\mathfrak{g})$, then $A$ is smooth. This follows from the result by Soergel 3 that the category of $A$-bimodules is equivalent to the category of left modules over some regular minimal primitive quotient of $U(\mathfrak{g} \oplus \mathfrak{g})$. Thus $A^{e}$ has finite global dimension and hence $A$ is smooth.

I wish to thank Andrea Solotar for bringing this error to my attention and for commenting on the present erratum.

\section{REFERENCES}

[1] S. Eilenberg, A. Rosenberg, and D. Zelinsky, On the dimension of modules and algebras, VIII, Nagoya Math. Journal 12 (1957), 71-93. MR 20:5229

[2] M. A. Farinati, A. Solotar, and M. Suarez-Alvarez, Hochschild homology and cohomology of generalized Weyl algebras, to appear.

[3] W. Soergel, The Hochschild cohomology of regular maximal primitive quotients of enveloping algebras of semisimple Lie algebras, Ann. Sci. École Norm. Sup. (4) 29 (1996), 535-538. MR 97e:17016

[4] M. Van den Bergh, Non-commutative homology of some three dimensional quantum spaces, J. K-theory 8 (1994), no. 3, 213-230. MR 95i:16009

[5] A relation between Hochschild homology and cohomology for Gorenstein rings, Proc. Amer. Math. Soc. 126 (1998), no. 5, 1345-1348. MR 99m:16013

Departement Wni, Limburgs Universitair Centrum, Universitaire Campus, Building D, 3590 Diepenbeek, Belgium

E-mail address: vdbergh@luc.ac.be 